\title{
REHABILITATION OF MUSCLE STRENGTH BY MEANS OF THE HUBER 360 PLATFORM IN LUMBAR RADICULOPATHY
}

\section{Carmen Liliana GHERGHEL ${ }^{1 *}$, Mariana CORDUN ${ }^{1}$, Ovidiu Cristian CHIRIAC ${ }^{1}$, Lucian Călin MARIN²}

\author{
${ }^{1}$ National University of Physical Education and Sport, Faculty of Kinetotherapy, Bucharest, Romania \\ 2 "Dr. Chiriac" Medical Rehabilitation Centre, Bucharest, Romania \\ *Corresponding author: carmen_gherghel@yahoo.com
}

DOI: $10.35189 /$ iphm.icpesk.2019.13

\begin{abstract}
For society, lumbosacralgia represents a financial problem and one of the main causes of disability; the average duration of absence from work because of a disorder in the lumbosacral region is 7 days. A lumbar stabilisation programme is a challenge for physical therapists in the case of patients with acute radiculopathy. The Huber 360 platform can be used within some specific programmes that aim at maintaining and improving health. The device is equipped with built-in force sensors and can be used in medical rehabilitation to both evaluate the patient and carry out the specific programmes. The purpose of this paper is to implement and verify the efficiency of distal proximal kinetotherapy achieved by using the Huber 360 platform as an alternative to classical rehabilitation methods. The study included 3 subjects, 1 woman and 2 men, who benefited from a rehabilitation protocol where both the evaluation and kinetotherapy programmes were performed using the Huber 360 platform. The results of the group emphasise an increase in maximum strength in a precise position at a certain moment and therefore it is noticed that the final strength values are balanced. The results of the study have shown that this approach is efficient in the functional treatment of compressive lumbar radiculopathy.
\end{abstract}

Keywords: radiculopathy, Huber 360, physical therapist.

\section{Introduction}

Low back pain is not a disease, but a symptom with multiple causes. The painful lumbar syndrome or lumbar radiculopathy (low back pain) is the most common type of muscular and skeletal condition, generally affecting the population of all ages and categories (Ionescu, 2007, p. 542).

Low back pain is the most frequent symptom among the working population, especially men. The highest prevalence is found in people aged 25 to 64 years. Low back pain is usually treated within two weeks, but the symptoms may take up to two months to disappear (Woolf \& Pleger, 2003). Also, 24\% to $80 \%$ of patients will have additional episodes when receiving treatment during the first year, and over three quarters will relapse at one point (Henschke et al., 2008). A small percentage, $2 \%$ up to $7 \%$ of patients, will develop chronic pain. Low back pain management can be expensive.

According to the literature ("Guideline for the Evidence-Informed Primary Care Management of Low Back Pain”, 2011), the medical rehabilitation treatment for radiculopathy during the acute period generally includes some recommendations. Thus, the patient must: remain active, continuing their normal activity within the limits of pain; avoid long bed rest (if the patient needs rest, this will be limited to only two days and no more than four); avoid accidents; take thermal (hot or cold) therapy but not directly applied for more than 15-20 minutes; stretching exercises; manual therapy - current orientations contraindicate manual manipulation in patients with severe or progressive neurological disorders; passive mobilisation in the form of traction should be avoided, because it is associated with significant side effects; McKenzie exercises are useful for the pain radiating below the knee; TENS (Transcutaneous Electrical Nerve Stimulation) is not recommended for treating acute pain in the lumbar area; if the symptoms persist for more than two weeks, non-invasive therapy shall be considered and will include joint mobility, flexibility and muscle strength programmes; if the symptoms persist for 6 weeks, a programme based on a multitask approach to lumbar pain shall be considered in the sub-acute period (4 to 8 weeks).

In general, the therapeutic intervention in lumbar discopathy is based on high disability scores, the neurological deficit and the patient's preference; all these criteria recommend the use of physical therapy. Compared to other nonsurgical management strategies, standard physical therapy is not associated with a significant difference in pain, disability or surgical intervention over 1 year. The best management strategy is unclear and further investigations are needed to examine proper sequencing and selection of treatment. (Thackeray, Fritz, Lurie, Zhao, \& Weinstein, 2017)

In this context, the medical recovery protocol proposed in this study is based on a kinetotherapy programme using the Huber 360 platform. 


\section{Material and Methods}

Various studies addressed the issue of therapeutic intervention in low back pain. Generally, it is considered that therapies with evidence of moderate effectiveness in chronic or sub-acute pain are: cognitive behavioural therapy, physical exercise, spinal manipulation and interdisciplinary rehabilitation. For acute back pain, the only therapy that has proven to be effective is superficial heat (Chou \& Huffman, 2007).

The purpose of our study is to include a physiotherapy programme performed using the Huber 360 multiaxial platform in the medical rehabilitation protocol for acute lumbar radiculopathy and highlight the obtained results.

The multiaxial Huber 360 platform with incorporated front sensors can be used for different purposes, thus being also useful in medical rehabilitation due to its ability to provide functional evaluation adapted to all types of patients. The Dynamic Posture Corrector offers precise and progressive training.

The platform allows customising the recovery progress of the patient and following up the results. This database can be connected to the $\mathrm{Wi}-\mathrm{Fi}$ and offers advantages in neural and muscular rehabilitation.

With the help of the Huber 360 platform, the treatment objectives are split into 4 fundamental movement parameters: muscle flexibility and joint mobility, dynamic strength, posture and balance.

The Huber 360 platform ensures safe joint mobilisation and quick pain reduction by using a wide range of exercises that lead to a rapid improvement in muscle flexibility and joint mobility, as well as the consolidation of the dynamic muscle strength.

In this paper, we shall approach the consolidation of dynamic muscle strength. We think it is important to mention that the monitor of the Huber 360 platform has an important feature that allows performing exercises in a very efficient way, which contributes to increasing muscle strength and improving coordination. The Huber 360 platform helps to strengthen superficial and deep muscles (Figure 1).

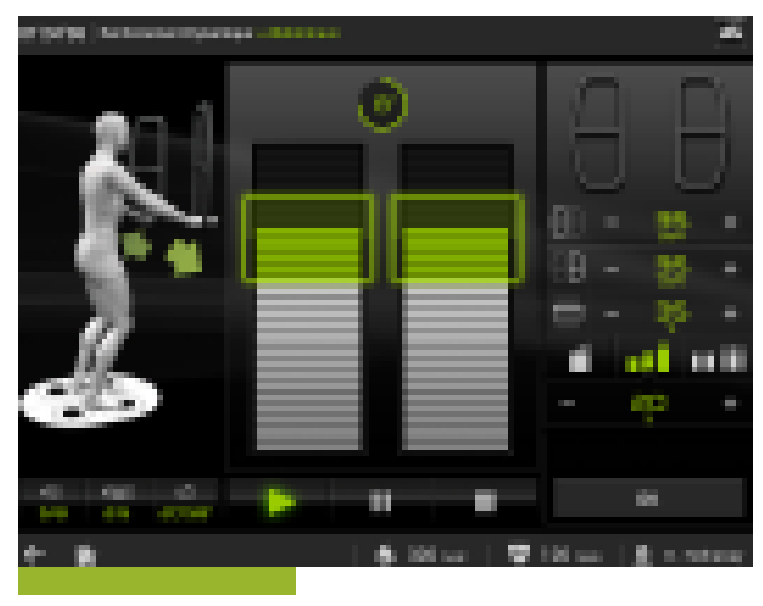

Figure 1. Muscle strengthening

The device can be used in hospitals, medical rehabilitation clinics or Wellness centres by physical therapists. It is an independent device that cannot be combined with other devices and cannot be used at home.

If low back pain, considered as the "pain of the century", is not treated, this will have a major impact on the patients' quality of life of life. For the purpose of functional recovery, we shall pursue several important objectives such as: reducing and combating lumbar pain, increasing strength in paravertebral, superficial and deep muscles, maintaining correct posture, as well as performing global movements. In parallel, it is important to consider the joint mobility of each vertebra in order to facilitate global range of motion through stretching exercises. With the multiaxial Huber 360 platform (an ideal functional instrument), muscle flexibility, joint mobility and muscle strength can be improved in comfort conditions for the patient through pain relief.

The study included three subjects, two males aged 35 and 65, respectively, and a female aged 25 . For the selection of these subjects, we used study inclusion and exclusion criteria. The inclusion criteria were: adults with lumbar pain or symptoms radiating in the lower limb with duration less than 6 weeks, "Yellow flags" - the presence of catastrophic thinking (there is no way for the patient to control pain, and its persistence will lead to disaster, etc.); the expectation that the pain will only get worse with work or activity; behaviours consisting in avoiding normal activity and prolonging rest; sleep disorders; feelings like stress and anxiety; work-related issues 
such as weak professional satisfaction and faulty relationships with superiors; prolonged periods of sabbatical leave (Braddom, 2015); pain intensity higher than 7 on a visual analogical scale. The exclusion criteria were represented by adults with lumbar pain or symptoms radiating in the lower limb with duration less than 6 weeks, "Red flags" - people under 18 years old with considerable pain, or the debut of pain in people over 55 ; history of violent trauma; pain without mechanical character; cancer history; systemic steroid consumption; medicine abuse; HIV infections or other forms of immunity compromise; unintentional weight loss; systemic pain, particularly signs of infection such as fever or nightly sweating; persistence of severe movement restriction or intense pain during minimum movement; structural deformities; difficulties while urinating; loss of anal sphincter tonus or faecal incontinence, saddle anaesthesia; progressive decrease of muscular strength or walking disorders; considerable morning stiffness; peripheral joint damage; uveitis, cutaneous rash, colitis, urethral leakages or other symptoms of rheumatologic conditions; inflammatory conditions such as spondylitis; hetero-collateral antecedents of rheumatologic conditions or structural abnormalities, pain intensity higher than 7 on a visual analogical scale.

Despite the considerable amount of studies on the role of physical therapy in acute radiculopathy, no consensus has been reached yet concerning the role of physical therapy in changing the evolution of acute radiculopathy. Saal, Saal and Yurth (1996) reported very good results following an aggressive non-surgical treatment (an active exercise programme associated with epidural steroid infiltrations) of the herniated lumbar disc with radiculopathy. This treatment protocol is the basis of several current exercise programmes used in herniated lumbar disc treatment with radiculopathy (Braddom, 2015, p. 960).

\section{Results}

The results of the group in the maximum strength tests for the left and right sides are summarised and presented in Table 1, where one can notice that most subjects have recorded an increase in maximum strength in a precise position at a certain moment for both stages (push/pull).

The result analysis shows that, for the first step (push), subject 1 (S1) does not show changes, but for the second (pull), the subject has recorded an improvement in maximum strength in a precise position at a certain moment with -2 daN (left) and -3 daN (right). Subject 2 (S2), according to the result analysis, shows an increase in maximum strength in a precise position at a certain moment for both stages, namely the left and right ones, and it can be noticed that the strength values are balanced in the two stages (left and right), the best result being obtained for the left pull stage, where the subject recorded an improvement of $-4 \mathrm{daN}$. In the final test, subject 3 (S3) managed to improve maximum strength in a precise position at a certain push time for both the left and right sides. For the pull stage, the strength recorded a drop of $-2 \mathrm{daN}$ for the left side and $-3 \mathrm{daN}$ for the right side.

Table 1. Maximum muscle strength for the left/right sides and the pull/push steps

\begin{tabular}{|c|c|c|c|c|c|c|}
\hline \multirow{2}{*}{ Action } & \multirow{2}{*}{$\begin{array}{r}\text { Maximum } \\
\text { Initial } \\
\end{array}$} & \multicolumn{2}{|c|}{$\begin{array}{l}\text { Muscle Strength (Left) } \\
(\mathrm{daN})\end{array}$} & \multicolumn{3}{|c|}{$\begin{array}{l}\text { Maximum Muscle Strength (Right) } \\
\text { (daN) }\end{array}$} \\
\hline & & Final & Difference & Initial & Final & Difference \\
\hline \multicolumn{7}{|l|}{ S1 } \\
\hline Pull (Pl) & 2 & 2 & 0 & 2 & 2 & 0 \\
\hline Push (Ps) & -2 & -4 & -2 & -2 & -5 & -3 \\
\hline \multicolumn{7}{|l|}{ S2 } \\
\hline Pull (Pl) & 11 & 14 & 3 & 13 & 15 & 2 \\
\hline Push (Ps) & -6 & -10 & -4 & -10 & -12 & -2 \\
\hline \multicolumn{7}{|l|}{ S3 } \\
\hline Pull (Pl) & 7 & 10 & 3 & 8 & 14 & 6 \\
\hline Push (Ps) & -9 & -7 & -2 & -9 & -6 & -3 \\
\hline
\end{tabular}




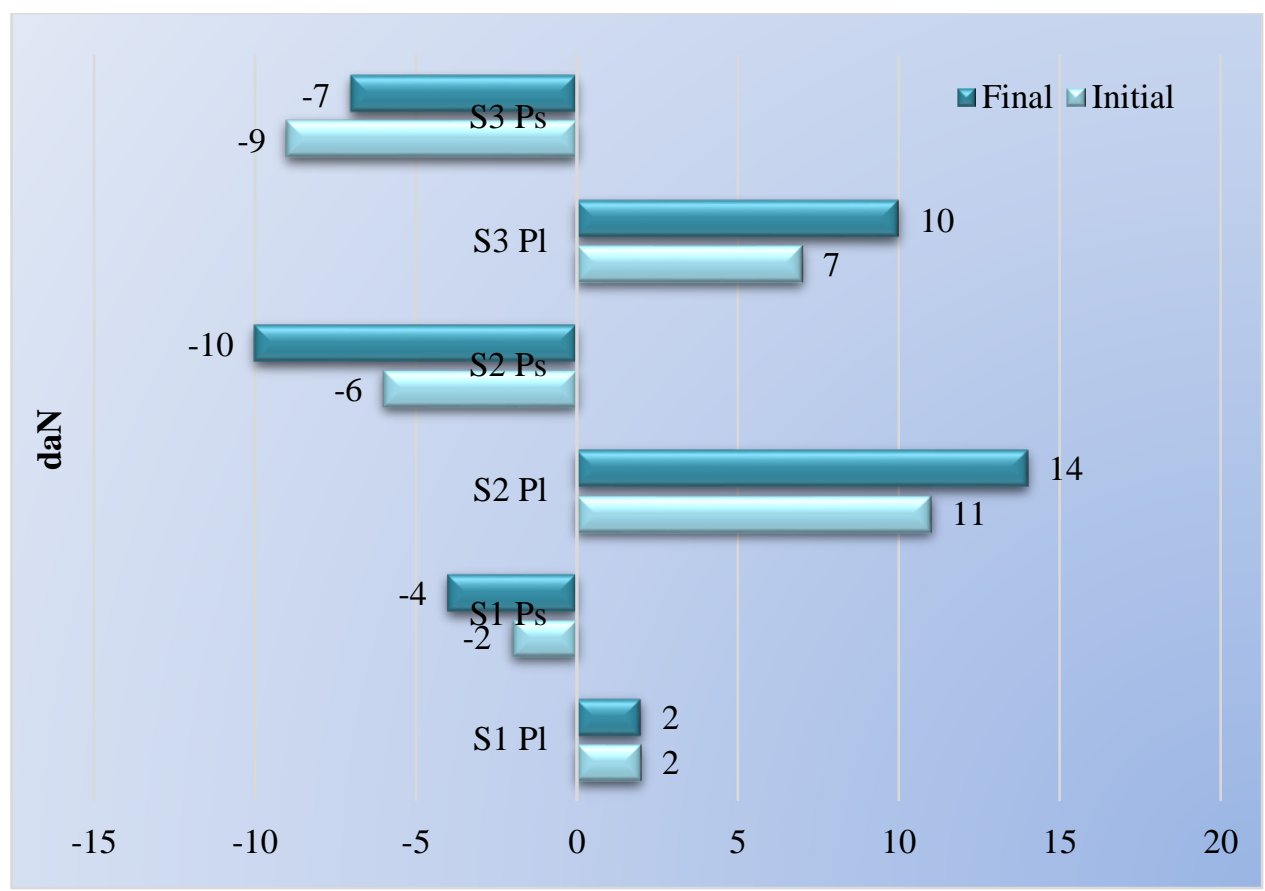

(S1 Ps - subject 1 push; S1 Pl - subject 1 pull; S2 Ps - subject 2 push; S2 P1 - subject 2 pull; S3 Ps - subject 3 push; S3 Pl - subject 3 pull)

Figure 2. Maximum muscle strength of the group for the left side

The results of the group for maximum strength (left side) in the two push and pull stages are shown in Figure 2 , which indicates that the maximum strength values are balanced in the two stages.

Low values can be seen in subject 1 , who, although aged 25 years old, has failed to improve strength, so we consider it to be due to the gender - female.

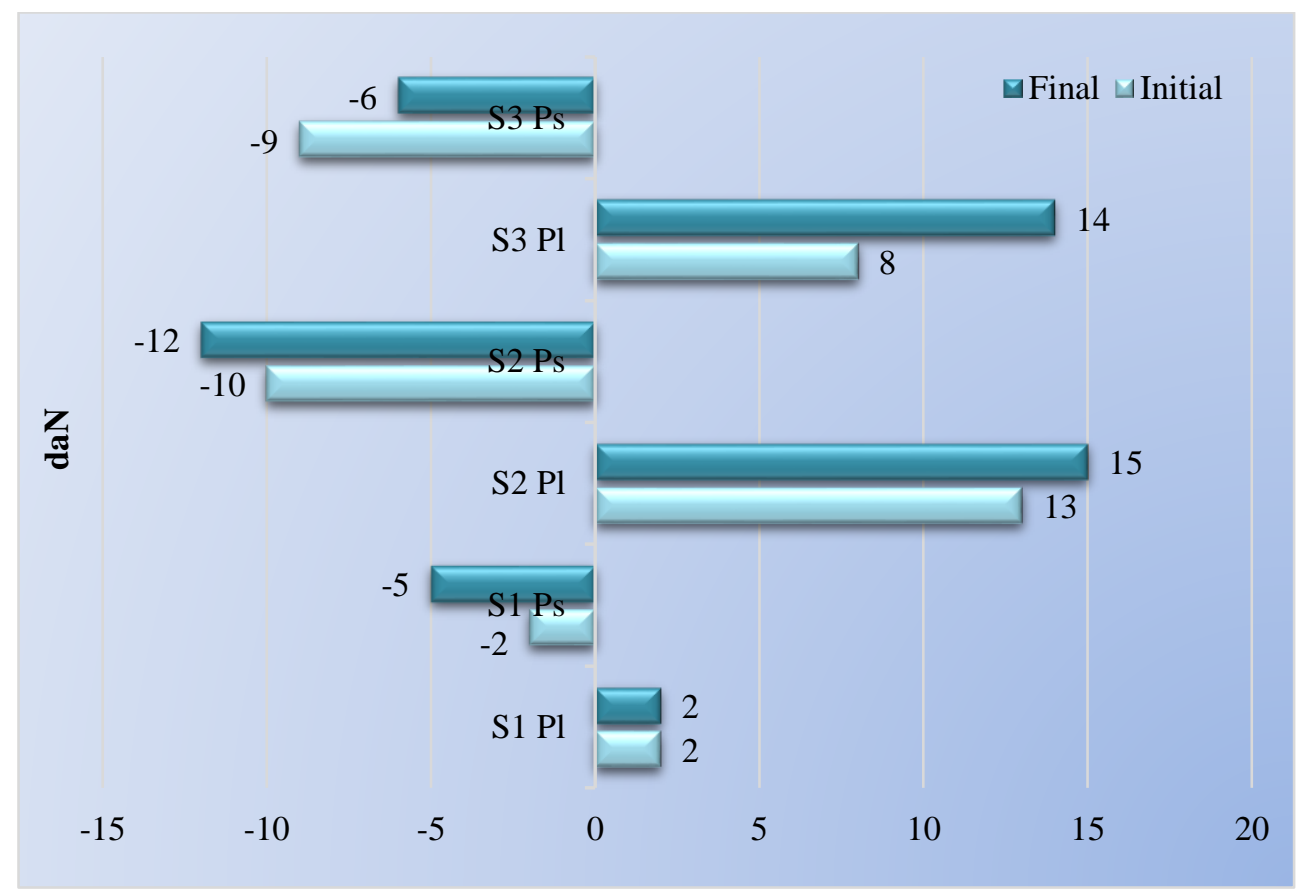

(S1 Ps - subject 1 push; S1 P1 - subject 1 pull; S2 Ps - subject 2 push; S2 P1 - subject 2 pull; S3 Ps - subject 3 push; S3 Pl - subject 3 pull)

Figure 3. Maximum muscle strength of the group for the right side 
The results of the group for maximum strength (right side) in the two push and pull stages are shown in Figure 3 , which indicates that the maximum strength values are balanced in the two stages.

It is highlighted the improvement of maximum strength in all subjects, subject 2 having an increase of 6 daN for the pull stage.

Low values can be noticed in subject 3 ( $-5 \mathrm{daN}$ for the push stage and $2 \mathrm{daN}$ for the pull stage), so we consider it to be due to age ( 68 years), however, he finally improved maximum strength by -3 daN.

\section{Conclusion}

Using the multiaxial Huber 360 platform in the acute lumbar radiculopathy protocol under the guidance of physical therapists will provide patients a way of working in safe conditions based on their physical and cognitive abilities, thus ensuring their neural and muscular rehabilitation. The Huber 360 platform can also be used within programmes for maintaining and improving health.

If low back pain, considered as the "pain of the century", is not treated, this will have a major impact on the patients' quality of life of life. With the multiaxial Huber 360 platform (an ideal functional instrument), muscle flexibility, joint mobility and muscle strength can be improved in comfort conditions for the patient through pain relief.

The results of the group in the maximum strength tests for the left and right sides in the two stages (push/pull) show an increase in maximum strength in a precise position at a certain time, and one can notice that the strength values are balanced, exceptions being explained by the old age or female gender of the subjects.

\section{References}

Braddom, R. L. (2015). Medicină fizică şi de reabilitare [Physical and rehabilitation medicine] (Ed. a IV-a). Bucureşti: Elsevier.

Chou, R., Huffman, L. H. (2007). Nonpharmacologic therapies for acute and chronic low back pain: A review of the evidence for an American Pain Society/American College of Physicians clinical practice guideline. Annals of Internal Medicine, 147(7), 492-504. https://doi.org/10.7326/0003-4819-147-7-200710020-00007

Guideline for the evidence-informed primary care management of low back pain ( $2^{\text {nd }}$ ed.). (2011). Retrieved from https://www.guidelinecentral.com/summaries/guideline-for-the-evidence-informed-primary-care-managementof-low-back-pain/\#section-420

Henschke, N., Maher, C. G., Refshauge, K. M., Herbert, R. D., Cumming, R. G., Bleasel, J., .. McAuley, J. H. (2008). Prognosis in patients with recent onset low back pain in Australian primary care: Inception cohort study. BMJ, 337(7662), 154-157. https://dx.doi.org/10.1136\%2Fbmj.a171

Ionescu, R. (2007). Esenţialul în reumatologie [Essentials of rheumatology] (Ed. a 2-a revizuită). Bucureşti: Editura Medicală.

Saal, J. S., Saal, J. A., \& Yurth, E. F. (1996). Nonoperative management of herniated cervical intervertebral disc with radiculopathy. Spine, 2l(16), 1877-1883. https://doi.org/10.1097/00007632-199608150-00008

Thackeray, A., Fritz, M. J., Lurie, J. D., Zhao, W., \& Weinstein, J. N. (2017). Nonsurgical treatment choices by individuals with lumbar intervertebral disc herniation in the United States: Associations with long-term outcomes. American Journal of Physical Medicine \& Rehabilitation, 96(8), 557-564. https://dx.doi.org/10.1097\%2FPHM.0000000000000685

Woolf, A. D., \& Pleger, B. (2003). Burden of major musculoskeletal conditions. Bulletin of the World Health Organization, 81(9), 646-656. Retrieved from https://www.ncbi.nlm.nih.gov/pmc/articles/PMC2572542/ 